\title{
A Best Cost of Null Controllability for the 1D Heat Equation by Strategic Zone Profile
}

\author{
Cheikh Seck (iD ${ }^{1,2}$ and Mame Libasse Laye Ane ${ }^{3}$ \\ ${ }^{1}$ Département de Mathématiques de la FASTEF ex ENS, de l'Université Cheikh Anta Diop de Dakar, Dakar, Senegal \\ ${ }^{2}$ Laboratoire d'Analyse Numérique et d'Informatique (LANI) de l'UFR SAT, BP 234, Université Gaston Berger, \\ Saint-Louis, Senegal \\ ${ }^{3}$ Département de Mathématiques et d'Informatique de la Faculté des Sciences et Techniques, \\ de l'Université Cheikh Anta Diop de Dakar, Dakar, Senegal
}

Correspondence should be addressed to Cheikh Seck; cheikh5.seck@ucad.edu.sn

Received 22 September 2021; Revised 28 October 2021; Accepted 10 November 2021; Published 29 November 2021

Academic Editor: Xiaolong Qin

Copyright (c) 2021 Cheikh Seck and Mame Libasse Laye Ane. This is an open access article distributed under the Creative Commons Attribution License, which permits unrestricted use, distribution, and reproduction in any medium, provided the original work is properly cited.

This study is the third step of a project on the null controllability of the 1D heat equation. First, we show a boundary and internal results of controllability by a new approach using a linear, continuous, and surjective operator built from the solution of the heat system. Second, we improve the minimum time of null controllability of the $1 \mathrm{D}$ heat equation by using the notion of strategic zone actuators. So, we managed to improve the minimal time of null controllability to the $1 \mathrm{D}$ heat equation. In this study, the best minimum cost of null controllability has been estimated for the $1 \mathrm{D}$ heat equation based on the minimum controllability time calculated in the second step.

\section{Introduction}

One of the objectives of the theory of the control of partial differential equations of evolution is to be interested in the way of acting on dynamic systems. The exact controllability of distributed systems has attracted a lot of interest in recent years. Thanks to the pioneer Fattorinni-Russel [1] and Lions $[2,3]$ who developed the HUM method (Hilbert Uniqueness Methods). It is based essentially on the properties of uniqueness of the homogeneous equation by a particular choice of controls and the construction of a Hilbert space and of a continuous linear application of this Hilbert space in its dual which is, in fact, an isomorphism that establishes exact internal or/and boundary controllability.

For hyperbolic problems, this method has given important results (Lions [2, 3], Niane [4], and Seck[5]).

Although when the controls have a small support (Niane [4], Guesmia [6], Glizer [7], Anguraj [8], and Seck et al [9-11]), it seems to be ineffective, even when for technical reasons the multiplier method does not give results.
As for the parabolic equations, there are the results of Russel [12] first. Later, G. Lebeau and el Robbiana [13] and Fursikov et al. [14] have proven with different methods which are very technical and long by using Carleman's Inequalities, the exact null controllability of the heat equation.

So, the harmonic method is also ineffective for this kind of equations.

In this study, we explain how results on the cost of null controllability of the small-time heat equation can be used to reduce the cost of control.

Indeed, more recently, Khodja et al. [15, 16] and Lissy [17], in particular, Tusnack-Tenebaum [18], have shown that there is a minimal time $T_{0}$ of controllability below which null controllability is not achievable for a parabolic operator. From the work of Khodja et al. [16] and Lissy and Guéye [17], a minimum cost of null controllability associated with the minimum time of null controllability of Russel [12] was calculated.

Indeed, motivated by the works of Khodja [15], Tucsnak [18], and Lissy $[17,18]$ on the null controllability of the heat equation and the work of El Jai $[19,20]$ on the controllability 
use of strategic zone actuators, we managed, in this work, to improve the minimum time of null controllability to the $1 \mathrm{D}$ heat equation.

However, the restrictions and difficulties to establish the inequality of coercivity of the parabolic operator require to seek other internal control methods.

Thus, a mixed method combining the moment methods and the notion of strategic profile was used to find a better minimal time of null controllability of the $1 \mathrm{D}$ heat equation.

Naturally, once this result had been obtained on the minimum time of null controllability, we began the calculations and estimates to find a minimum control cost linked to this minimum time. This is how we established a best minimum cost of null controllability of the heat equation with an additional assumption on the strategy profile function.

These results open up broad prospects in this domain. Particularly, on semianalytical systems, the piecewise temperature-time distributions in solid bodies of regular shape were affected by a uniform surface heat flux using the line method (MOL) and the eigenvalue method. This method is also used in the numerical analysis of unstable thermal conduction in regular solid bodies including natural convection towards neighboring fluids, as well as for the calculation of spatio-temporal temperatures in simple bodies with cooling by thermal radiation using the method of digital lines.

\section{Preliminary}

2.1. Notations and Definition. Let $T>0$; the sequences of reals $\Lambda=\left(\lambda_{k}\right)_{k \geq 1}$; let us define now the setting that we will deal in the sequel and assume that

$$
\sum_{k=1}^{+\infty} \frac{1}{\lambda_{k}}<+\infty
$$

Definition 1. The condensation index of sequences $\Lambda=\left(\lambda_{k}\right)_{k \geq 1}$ is defined as

$$
I(\Lambda)=\lim _{k \rightarrow+\infty} \sup \frac{-\ln \left|E^{\prime}\left(\lambda_{k}\right)\right|}{\lambda_{k}},
$$

where the function $E$ is defined by

$$
E(x):=\prod_{k=1}^{+\infty}\left(1-\frac{x^{2}}{\lambda_{k}^{2}}\right) \text {. }
$$

2.2. Concept of Strategic Zone Actuators. A function $\mu: I \longrightarrow \overline{\mathbb{R}}$ square integrable is said strategic if it verifies for all $y_{0} \in L^{2}(I)$, the solution $y$ of the heat equation:

$$
\begin{aligned}
& \begin{cases}y_{t}(t, x)-\partial_{x x} y(t, x)=0, & \text { in } Q_{T}=[0,+\infty[\times I, \\
\gamma y(t, x)=0, & \text { in } \Sigma_{T}=[0,+\infty[\times \partial I \\
y(0)=y_{0} & \text { in } I,\end{cases} \\
& \forall t>0, \\
& \int_{I} \mu(x) y(t, x) \mathrm{d} x=0
\end{aligned}
$$

Let $I=] 0, \pi$ [ be an interval of $\mathbb{R}$; let $A$ be the operator defined by

$$
D(A)=\left\{y \in \frac{H_{0}^{1}(I)}{-\partial_{x x} y} \in L^{2}(I),\right\} A y=-\partial_{x x} y, \quad \forall y \in D(A) .
$$

According to the spectral theory, see Lions [3], A admits a Hilbertian base of $L^{2}(I)$ of eigenfunctions $\left(w_{k}\right)_{k \geq 1}$ whose associated eigenvalues are $\left(\lambda_{k}\right)_{k \geq 1}$ rows in the ascending direction, where

$$
\left\{\begin{array}{l}
w_{k}(x)=\sqrt{\frac{2}{\pi}} \sin k x \\
\lambda_{k}=k^{2}
\end{array}\right.
$$

Proposition 1. There are strategic actuators with support contained in any interval ] $a, b[$ such that $0<a<b<\pi$.

Proof. We can first notice that $\mu$ is strategic if and only if $\forall k \in \mathbb{N}^{*}, \mu_{k} \neq 0$.

Let $a, b \in] 0, \pi\left[\right.$ such that $a<b$ and posing that $\mu=\chi_{] a, b}$. Then, we have

$$
\begin{aligned}
\mu_{k} & =\int_{0}^{\pi} \chi_{] a, b[}(x) \frac{\sqrt{2}}{\sqrt{\pi}} \sin (k x) \mathrm{d} x \\
& =-\frac{\sqrt{2}}{k \sqrt{\pi}}[\cos k(b)-\cos k(a)] \\
& =-\frac{\sqrt{2}}{k \sqrt{\pi}} 2 \sin \left(\frac{k(b-a)}{2}\right) \sin \left(\frac{k(b+a)}{2}\right) .
\end{aligned}
$$

We have $\mu_{k}=0$ if and only if

$$
\begin{cases}\frac{k(b-a)}{2}=l, & l \in \mathbb{Z}, \\ \frac{k(b+a)}{2}=r, & r \in \mathbb{Z} .\end{cases}
$$

Therefore, for that $\mu_{k} \neq 0$, it is sufficient that $b-a \notin \mathbb{Q}$ and $b+a \notin \mathbb{Q}$.

So, if we take $a \in \mathbb{Q}$ and $b=a+r$, where $r \notin \mathbb{Q}$, then $\mu=\chi_{] a, b[}$ is strategic.

Remark 1. Obviously, other strategic actuators can be built without great difficulty, see the work of Jai et al. $[19,20]$ and Seck and Ane [11].

\subsection{Reminders on the Minimal Time of Null Controllability for} the $1 D$ Heat Equation. Let

$$
\begin{cases}y_{t}-y_{x x}=f(x) u(t), & \text { in }[0 ; T[\times[0 ; \pi[, \\ y(0 ; \cdot)=y^{0}, & \text { in }[0 ; \pi[, \\ y(\cdot ; 0)=y(0 ; \pi)=0, & \text { in }[0 ; T[,\end{cases}
$$

where $y^{0} \in L^{2}\left(\left[0 ; \pi[), u \in L^{2}\left(\left[0 ; T[)\right.\right.\right.\right.$, and $f \in H^{-1}([0 ; \pi[)$. 
We know that system (9) admits a unique solution $y \in\left(C^{0}\left[0 ; T\left[;\left[0 ; \pi[) \cap\left(L^{2}\left[0 ; T\left[; H_{0}^{1}[0 ; \pi[)\right.\right.\right.\right.\right.\right.\right.$, see the work of Lions [3].
Verifying $\exists C>\left(0 / \forall y^{0}\right) \in L^{2}\left[0 ; \pi\left[, \forall f \in H^{-1}[0 ; \pi[\right.\right.$, and $\forall v \in L^{2}[0 ; \pi[$, we have

$$
\|y\|_{\left(C ^ { 0 } \left[0 ; T\left[; L^{2}[0 ; \pi[)\right.\right.\right.}+\|y\|_{\left(L ^ { 2 } \left[0 ; T\left[; H_{0}^{1}[0 ; \pi[)\right.\right.\right.} \leq C\left(\|y\|_{L^{2}[0 ; \pi[}+\|f\|_{H^{-1}[0 ; \pi[} \cdot\|v\|_{L^{2}[0 ; T]}\right) .
$$

Let

$$
\begin{aligned}
I_{k}(f) & :=-\frac{\log (|f|)}{k^{2}} \\
T_{0} & :=\lim _{k \longrightarrow+\infty} \sup I_{k}(f) \text { in }[0,+\infty[.
\end{aligned}
$$

We know from the work of Russel and Fatorrini [12], Lebeau and et Robbiano [13], and Fursikov et al. [14] that there exists a minimum time of control of the $1 \mathrm{D}$ heat equation.

So,

(1) System (9) is null controllable at any time $T>T_{0}$

(2) System (9) is not null controllable at any time $T<T_{0}$

Example 1. Consider the following examples:

Example 1: if $f(x)=\delta\left(x_{0}\right) \in H^{-1}[0 ; \pi[, \quad$ with $x_{0}$ in $[0 ; \pi[$,

$$
T_{0}\left(x_{0}\right)=\lim _{k \longrightarrow+\infty} \sup \frac{-\log \left|\sin \left(k x_{0}\right)\right|}{k^{2}} .
$$

$\forall x_{0}$ in $\left[0 ; \pi\left[; T_{0}\left(x_{0}\right)=0\right.\right.$.

$\forall \tau$ in $\left[0 ; \infty\left[;\left\{\left(x_{0}\right) \in\left(\left[0 ; \pi\left[/ T_{0}\left(x_{0}\right)\right)=\tau\right\}\right.\right.\right.\right.$ is dense in $[0 ; \pi[$.

Example 2: if the profile $f$ defined for any $t>0$ over an interval $[0, L]$ by

$$
f_{[[0, L]}(x)= \begin{cases}\exp \left(-\frac{t}{1-x^{2}}\right), & \text { if }|x|<1, \\ 0, & \text { else, }\end{cases}
$$

then $f$ is a function with strong rapid decay and by setting $C_{H}(T)$, the cost of the control, at the terminal instant. Let us pose

$$
\alpha^{+}(H):=\lim \sup _{T \rightarrow 0} \frac{T \cdot \ln \left(C_{H}(T, L)\right)}{L^{2}} .
$$

Also,

$$
\alpha^{-}(H):=\lim \inf _{T \longrightarrow 0} \frac{T \cdot \ln \left(C_{H}(T, L)\right)}{L^{2}} .
$$

We show by simple calculations that $\alpha^{-}>0$ and $\alpha^{+}<+\infty$.

In particular, if $L=\pi$, then $C_{H}(T) \simeq e^{C \pi^{2}}$ with $T>T_{0}$ but small, where $C$ is the constant defined above.
In the case $T>T_{0}$, for $\forall y_{0}$ in $L^{2}([0 ; \pi[)$, there exists a unique optimal control $u_{\text {opt }} \in L^{2}\left(\left[0 ; T[)\right.\right.$ bringing $y^{0}$ to 0 , see the work of Lissy et al. [18].

The map $\mathscr{L}: y^{0} \mapsto u_{\text {opt }}$, being linear continuous.

Definition 2. The norm of this operator $\mathscr{L}$ is called the optimal null control cost at time $T$ designated by $C_{H}(T)$.

So, by Definition 2, $C_{H}(T)$ is infinimum of the constants $C>0$ such that $\forall y^{0}$ in $L^{2}\left[0 ; \pi\left[; \exists u\right.\right.$ driving $y^{0}$ to 0 at time $T$ with

$$
\|u\|_{L^{2}[0 ; T]} \leq C\left\|y^{0}\right\|_{L^{2}[0 ; \pi[} .
$$

What is the behavior of $C_{H}(T)$ when $T \longrightarrow T_{0}^{+}$?

One could expect that the cost is of the form

$$
\frac{C\left(T_{0}\right)}{T-T_{0}} \quad \text { as } T \longrightarrow T_{0}^{+} \text {. }
$$

In the work of Seck et al. [11], we successful find a better control time (noted $T_{0}^{\mu}$ ) compared to that proposed by Khodja et al. [16], $T_{0}$, which led us to the main result of this work.

\section{Main Result on the Minimum Cost Linked to the Minimum Time Null Controllability of $1 D$ Heat Equation}

\subsection{Fundamental Lemma}

Lemma 1. If $\mu$ is a strategic actuator on $[0, \pi], u(\cdot)$ is a control and $T>0$ is strictly positive and real; for all $y_{0} \in F_{T}^{*}$, there exist $\left.\left.\beta \in L^{2}(] 0, T\right]\right)$ and $T_{0}^{\mu}<T$ such that if $y$ is solution of

$$
\begin{cases}y_{t}-\partial_{x x} y=\beta(t) \mu(x) u(t) & \text { in }] 0, T[\times I, \\ \gamma y=0, & \text { in }] 0, T[\times \partial I, \\ y(0)=y_{0}, & \text { in } I,\end{cases}
$$

then $y(T)=0$.

For proof, see the work of Seck et al. in [11].

Consider the heat equation with an internal strategic zone profile $\mu(\cdot)$ and a, internal control $u(\cdot)$ defined by

$$
\left\{\begin{array}{l}
\left.y_{t}-\partial_{x x} y=\beta(t) \mu(x) u(t) \quad \text { in } Q_{T}=\right] 0,+\infty[\times I, \\
y(0 ; t)=y(\pi ; t)=0 \quad \text { in }] 0, T[ \\
y(0)=y_{0} .
\end{array}\right.
$$


Let $B=\beta(t) u(t)$ be a linear control operator; then, the previous equation (19) becomes

$$
\left\{\begin{array}{l}
y_{t}-\partial_{x x} y=B \mu(x) \text { in } Q_{T}, \\
y(0 ; t)=y(\pi ; t)=0, \\
y(0)=y_{0} .
\end{array}\right.
$$

In the sequel of this study, the minimum time null controllability is denoted by $T_{0}^{\mu}$.

Recall that $T_{0}^{\mu}$ is calculated and defined in the work of Seck et al. [11] as follows:

$$
T_{0}^{\mu}=\lim \sup _{k \geq 1} \frac{\log \left(1 / \beta_{k}\right)+\log (1 / \mu(x))+\log \left(1 /\left|E^{\prime}\left(k^{2}\right)\right|\right)}{k^{2}} .
$$

From the minimal time of null controllability of system (19), then we obtain $T_{0}^{\mu}=T_{0}+\limsup _{k \geq 1}\left(\log (1 / \mu(x)) / k^{2}\right)$ with $T_{0}=\limsup _{k \geq 1}\left(\left(\log \left(1 / \beta_{k}\right)+\log \left(1 /\left|E^{\prime}\left(k^{2}\right)\right|\right)\right) / k^{2}\right)$, where $T>T_{0}^{\mu}$ and $\mu(x)>1$.

Theorem 1 (main theorem). Let $\Psi: \mathbb{R}_{+}^{*} \longrightarrow \mathbb{R}_{+}^{*}$ be an increasing function verifying morever $\Psi(x) \longrightarrow+\infty$ as $x \longrightarrow+\infty$ and $\mu(x)>1$ ( $\mu$ a strategic profile); $\forall T_{0}^{\mu} \in[0 ; \infty[;$ there exists $f:=B \mu \in\left(H^{-1}\left([0 ; \pi[)) /\left(\forall T \longrightarrow T_{0}^{\mu}\right)\right.\right.$ :

$$
C_{H, \mu}(T) \geq \frac{1}{\sqrt{T}} \Psi\left(\frac{1}{T-T_{0}^{\mu}}\right),
$$

where $C_{H, u}(T)$ is the infinimum of the constants $C>0$ such that $\forall y^{0}$ in $L^{2}\left[0 ; \pi\right.$ [, there is a control $u$ driving $y^{0}$ to 0 at time $T$.

Remark 2. This theorem means that the cost of the control can increase arbitrarily fast as $T \longrightarrow T_{0}^{\mu}$.

This can be explained by the fact that, contrary to the usual case, the cost of the control depends not only on the behavior of $I_{k}(f)$ at infinity but also on how it differs from its limit superior $T_{0}^{\mu}$.

Proof. $C$ will be a positive constant independent of $T$.

Let us fix $T_{0}^{\mu}$ in $\left[0 ; \infty\left[\right.\right.$, and we consider $T>T_{0}^{\mu}$, and let $n \in \mathbb{N}^{*}$ be chosen later.

We define $y^{0}$ in $L^{2}\left(\left[0 ; \pi[)\right.\right.$ as follows: $y^{0}(x)=\sin (n x)$.

One readily verifies that there exists some positive constant $C$ such that

$$
\left\|y^{0}\right\|_{L^{2}[0 ; \pi[} \leq C
$$

We consider the optimal control $u$ associated to this initial condition, which verifies by definition and estimates the control:

$$
\|u\|_{L^{2}[0 ; T[} \leq C_{H}(T)\left\|y^{0}\right\|_{L^{2}[0 ; \pi[} \leq C_{H} C .
$$

By the moments' method, we obtain $\forall k \in \mathbb{N}^{*}$ :

$f(k) \int_{0}^{T} u(t) \exp \left(k^{2} t\right) \mathrm{d} t=-\int_{0}^{\pi} \sin (n x) \sin (k x) \mathrm{d} x$.

Applying for $k=n$, we have

$$
\begin{aligned}
f(n) \int_{0}^{T} u(t) \exp \left(n^{2} t\right) \mathrm{d} t & =-\int_{0}^{\pi} \sin ^{2}(n x) \mathrm{d} x, \\
\int_{0}^{\pi} \sin ^{2}(n x) \mathrm{d} x & =\left[\frac{n x-\sin (n x) \cos (n x)}{2 n}\right]_{0}^{\pi} .
\end{aligned}
$$

Now,

$\int_{0}^{\pi} \sin ^{2}(n x) \mathrm{d} x=\frac{\pi}{2} \Rightarrow \int_{0}^{T} u(t) \exp \left(n^{2} t\right) \mathrm{d} t=\frac{-\pi}{2 f(n)}$.

We know that

$$
\left|\int_{0}^{T} u(t) \exp \left(n^{2} t\right) \mathrm{d} t\right|=\frac{\pi}{2|f(n)|}
$$

Also,

$$
\begin{aligned}
\left|\int_{0}^{T} u(t) \exp \left(n^{2} t\right) \mathrm{d} t\right| & \leq\left|\int_{0}^{T} u(t) \mathrm{d} t\right|\left|\int_{0}^{T} \exp \left(n^{2} t\right) \mathrm{d} t\right| \\
& \leq\left[\frac{\exp \left(n^{2} t\right)}{n^{2}}\right]_{0}^{T}\left|\int_{0}^{T} u(t) \mathrm{d} t\right| \\
& \leq \frac{\exp \left(n^{2} T\right)}{n^{2}} \int_{0}^{T}|u(t)| \mathrm{d} t \\
& \leq \exp \left(n^{2} T\right) \int_{0}^{T}|u(t)| \mathrm{d} t \\
\Rightarrow \frac{\pi}{2|f(n)|} & \leq \exp \left(n^{2} T\right) \int_{0}^{T}|u(t)| \mathrm{d} t .
\end{aligned}
$$
that

Applying the Cauchy-Schwarz inequality, we deduce

$$
\frac{\pi}{2|f(n)|} \exp \left(-n^{2} T\right) \leq \sqrt{T}\|u\|_{L^{2}[0 ; T[}
$$

So, we have

$$
\begin{aligned}
C \times C_{H}(t) & \geq\|u\|_{L^{2}[0 ; T[} \\
& \Rightarrow C_{H}(T) \geq \frac{1}{C}\|u\|_{L^{2}[0 ; T[} \geq \frac{1}{C} \frac{\pi}{2|f(n)|} \frac{\exp \left(-n^{2} T\right)}{\sqrt{T}} \\
C_{H}(T) & \geq \frac{C}{|f(n)|} \frac{\exp \left(-n^{2} T\right)}{\sqrt{T}} .
\end{aligned}
$$

Now, let us consider any positive and increasing function $\psi: \mathbb{R}_{+}^{*} \longrightarrow \mathbb{R}_{+}^{*}$ such that $\psi(x) \longrightarrow \infty$ when $x \longrightarrow \infty$.

Such a function is necessarily bijective and we call $\psi^{-1}$ its inverse.

Let us consider $\left(f_{n}\right)_{n \in \mathbb{N}^{*}}$ defined by

$$
f(n):=\exp \left(-n^{2}\left(T_{0}^{\mu}+\frac{1}{\psi^{-1}\left(n^{2}\right)}\right)\right) \text { in } L^{2}\left(N^{*}\right),
$$

so that we have the condensation index (see the works of Khodja [16] and Tusnack [19]) defined by 


$$
\begin{aligned}
I_{n}(f)= & -\frac{\log (f(n))}{n^{2}} \\
& \cdot \frac{-\log \exp \left(-n^{2}\left(T_{0}^{\mu}+\left(1 / \psi^{-1}\left(n^{2}\right)\right)\right)\right.}{n^{2}} \\
= & \frac{n^{2}\left(T_{0}^{\mu}+\left(1 / \psi^{-1}\left(n^{2}\right)\right)\right)}{n^{2}} \\
= & T_{0}^{\mu}+\left(1 / \psi^{-1}\left(n^{2}\right)\right) .
\end{aligned}
$$

It is clear that (see the work of Seck [11])

$$
T_{0}^{\mu}=\lim \sup _{k \geq 1} \frac{\log \left(1 / \beta_{k}\right)+\log (1 / \mu(x))+\log \left(1 /\left|E^{\prime}\left(k^{2}\right)\right|\right)}{k^{2}},
$$

i.e.,

$$
T_{0}^{\mu}=T_{0}+\lim \sup _{k \geq 1} \frac{\log (1 / \mu(x))}{k^{2}}
$$

where

$$
T_{0}=\lim \sup _{k \geq 1} \frac{\log \left(1 / \beta_{k}\right)+\log \left(1 /\left|E^{\prime}\left(k^{2}\right)\right|\right)}{k^{2}},
$$

where the function $E$ is defined by

$$
E(x):=\prod_{k=1}^{+\infty}\left(1-\frac{x^{2}}{\lambda_{k}^{2}}\right)
$$

since $\psi^{-1}\left(n^{2}\right) \longrightarrow+\infty$.

Then, we have to thank the works of Khodja [15] and Seck $[10,11]$ :

$$
C_{H}(T) \geq \frac{C}{\sqrt{T}} \exp \left(n^{2}\left(T_{0}^{\mu}-T+\frac{1}{\psi^{-1}\left(n^{2}\right)}\right) .\right.
$$

Let us explain how to choose $n$, and we assume that $T$ is close enough to $T_{0}^{\mu}$.

Now, we choose $n$ in such a way that

$$
\frac{1}{2\left(T-T_{0}^{\mu}\right)} \geq \psi^{-1}\left(n^{2}\right) \geq \frac{1}{4\left(T-T_{0}^{\mu}\right)},
$$

which is always possible (at least for $T$ close enough to $T_{0}^{\mu}$ ), since $\psi^{-1}$ is increasing and goes to $+\infty$ at $+\infty$.

Indeed,

$$
\begin{aligned}
\psi \circ \psi^{-1}\left(n^{2}\right) & \geq \psi\left(\frac{1}{4\left(T-T_{0}^{\mu}\right)}\right) \\
\frac{1}{2\left(T-T_{0}^{\mu}\right)} \geq \psi^{-1}\left(n^{2}\right) \geq \frac{1}{4\left(T-T_{0}^{\mu}\right)} & \\
\Rightarrow n^{2} & \geq \psi\left(\frac{1}{4\left(T-T_{0}^{\mu}\right)}\right) \\
& \Rightarrow \frac{C}{\sqrt{T}} \exp \left(n^{2}\left(T_{0}^{\mu}-T+\frac{1}{\psi^{-1}\left(n^{2}\right)}\right)\right) \geq \frac{C}{\sqrt{T}} \exp \left(\psi\left(\frac{1}{4\left(T-T_{0}^{\mu}\right)}\right)\left(T_{0}^{\mu}-T+\frac{1}{\psi^{-1}\left(n^{2}\right)}\right)\right. \\
\psi^{-1} & \longrightarrow+\infty \text { so } \frac{1}{\psi^{-1}\left(n^{2}\right)} \longrightarrow 0 .
\end{aligned}
$$

Consequently,

$$
C_{H}(T) \geq \frac{C}{\sqrt{T}} \exp \left(\left(T_{0}^{\mu}-T\right) \psi\left(\frac{1}{4\left(T-T_{0}^{\mu}\right)}\right)\right) .
$$

One then easily obtains the desired result by choosing $\psi$ in such a way that

$$
\Psi(x)=C \exp \left(\frac{1}{x} \psi\left(\frac{x}{4}\right)\right) \Leftrightarrow \psi(x)=4 x \log \left(\frac{\Psi(4 x)}{C}\right) .
$$

Because it is clear that if $\Psi$ is positive, increasing, and converge to $+\infty$ at $+\infty$, then $\psi$ is well defined at least for large enough $x$ which is sufficient for our purpose.

Remark 3. The assumptions on the function $\Psi$ make it possible to bypass the coercivity of the parabolic operators, which is, moreover, difficult to establish for these kinds of operators 


\section{Conclusion and Perspectives}

The theory of the control of distributed systems has experienced a meteoric rise in recent years, notably with the team of Lions and Coron [22] and, more recently, with Ammar Khodja, Tucsnak, and Guesmia. In particular, the controllability of the heat equation has been established since the mid-90s by Lebeau-Robbiano and Fursikov-Imanuvilov. In all these works and others more recent works of TucsnackTenenbaum and Khodja et al., there is always a time $T_{0}$ from which the control is realizable (below $T_{0}$, controllability is not feasible). Our aim was to find a better minimum time $T_{0}^{\mu}$ to carry out this control (knowing that we cannot control the heat equation all the time). Indeed, if $T_{0}^{\mu}<T_{0}$, then the cost $C_{H}^{\mu}(T)$ is better than $C_{H}(T)$ (this is our main result).

The other objective of this work was to solve an optimal control problem. Thus, our modest contribution is improving the time (smaller time) and hence reducing the cost to achieve the control of the heat equation over a small support interval. Indeed, we knew that there is no uniqueness of the control profile bringing the system of the initial condition $y_{0}$ to the final state (the set of strategic profiles is a closed affine subspace: we can naturally choose a norm control minimal as being the projection of 0 on this convex).

This method is no longer operative when the interval support is large or if there is a nonlinear term in the system.

We plan, in the near future, to generalize this result to the Schrödinger equation, to transport-type equations, and to linear dispersive parabolic systems.

\section{Data Availability}

The data used to support the findings of this study are available from the corresponding author upon request.

\section{Conflicts of Interest}

The authors declare that they have no conflicts of interest.

\section{References}

[1] H. O. Fattorrini and D. L. Russell, "Exact controllability theorems for linear parabolic equations in one space dimension," Archive for Rational Mechanics and Analysis, vol. 43, no. 4, pp. 272-292, 1971.

[2] J.-L. Lions, "Contrôlabilité exacte, perturbations et stabilisation de systèmes distribués," Tome 2, Recherches en Mathématiques Appliquées, Research in Applied Mathematics, vol. 9, 1988.

[3] J.-L. Lions, "Contrôlabilité exacte, perturbations et stabilisation de systèmes distribués," Tome 1, Recherches en Mathématiques Appliquées, Research in Applied Mathematics, vol. 8, 1988.

[4] M. T. Niane, "Contrôlabilité spectrale élargie des systèmes distribués par une action sur une partie analytique arbitraire de la frontière," Comptes Rendus de l'Académie des Sciences, vol. 309, no. 1, pp. 335-340, 1989.

[5] C. Seck, "Exact controllability of the heat equation by temporarily strategic actuators borders," Journal of Mathematics Research, vol. 11, no. 6, p. 53, 2019.
[6] A. Guesmia, M. Kafini, and N. E. Tatar, "General stability results for the translational problem of memory-type in porous thermoelasticity of type III," Journal of Nonlinear Functional Analysis, vol. 2020, no. 1, p. 49, 2020.

[7] V. Y. Glizer, "Euclidean space controllability conditions and minimum energy problem for time delay systems with a high gain control," Journal of Nonlinear and Variational Analysis, vol. 2, no. 1, pp. 63-90, 2018, https://jnva.biemdas.com.

[8] A. Anguraj and K. A. Ramkumar, "Global existence and controllability to a stochastic integro-differential equation with Poisson jumps," Communications in Optimization Theory, vol. 2018, p. 22, 2020.

[9] C. Seck, L. Ane, and A. Sène, "Boundary exact controllability of the heat equation in 1D by strategic actuators and a linear surjective compact operator," Applied Mathematics, vol. 11, no. 10, pp. 991-999, 2020.

[10] C. Seck, A. Sène, and M. Niane, "Estimates related to the extended spectral control of the wave equation," Journal of Mathematics Research, vol. 10, no. 4, p. 156, 2018.

[11] C. Seck and M. L. L. Ane, "Minimal time of null controllability for the $1 \mathrm{D}$ heat equation by a strategic zone profile," Journal of Applied Mathematics and Physics, vol. 09, no. 07, pp. 1707-1717, 2021.

[12] D. L. Russel and H. O. Fattorini, "Exact controllability for linear parabolic equation in one space dimension," Archive for Rational Mechanics and Analysis, vol. 4, pp. 272-292, 1971.

[13] G. Lebeau and L. et Robbiano, Contrôle exact de l'équation de la Chaleur, Prépublications Université Paris-Saclay, Orsay, France, 1994.

[14] A. V. Fursikov, et Imanuvilov, and O. Yu, Controllability of Evolution Equations, Séoul National University, Seoul, South Korea, 1996.

[15] F. Ammar Khodja, A. Benabdallah, M. González-Burgos, and L. de Teresa, "Minimal time for the null controllability of parabolic systems: the effect of the condensation index of complex sequences," Journal of Functional Analysis, vol. 267, no. 7, pp. 2077-2151, 2014.

[16] F. A. Khodja, A. Benabdallah, M. Gonzàlez-Burgo, and L. D. Teresa, "Minimal time for the null controllability of parabolic systems: the effect of the condensation index of complex sequences," 2013, https://hal.archives-ouvertes.fr/ hal-00918596.

[17] P. Lissy and M. Guéye, "Singular optimal control of a 1-D parabolic-hyperbolic," Degenerate Equation, https://hal. archives-ouvertes.fr/hal-01240264, 2015.

[18] P. Lissy, "A link between the cost of fast controls for the 1-D heat equation and the uniform controllability of a 1-D transport-diffusion equation," Comptes Rendus Mathematique, vol. 350, no. 11-12, pp. 591-595, 2012.

[19] G. Tenenbaum, M. Tucsnak, and Gerald, "New blow-up rates for fast controls of Schrödinger and heat equations," Journal of Differential Equations, vol. 243, no. 1, pp. 70-100, 2007.

[20] A. El Jai, "Quelques problèmes de contrôle propres aux systèms distribués," Annals of University of Craiova, Math. Comp. Sci. Ser.vol. 30, pp. 137-153, 2003.

[21] A. El Jai, “Analyse régionale des systèmes distribués," ESAIM: Control, Optimisation and Calculus of Variations, vol. 8, pp. 663-692, 2002.

[22] J. M. Coron, "Control and nonlinearity," Mathematical Surveys and Monographs, American Mathematical Society, vol. 136, p. 2007, Providence, RI, USA, 1976. 